BRX-TH-338

\title{
CP-Violating Yukawa Couplings in the Skyrme Model and the Neutron Electric Dipole Moment
}

\author{
Harold A. Riggs and Howard J. Schnitzer \\ Department of Physics, Brandeis University \\ Waltham, MA 02254
}

November 1992

\begin{abstract}
We argue that the large- $N_{c}$ behaviour of the Yukawa couplings in the Skyrme model involves issues more subtle than the vanishing of linear fluctuations needed for classical stability of the skyrmion. The chiral fluctuations about the skyrmion must be quantized in order to reach a conclusion. An improved quantization procedure allows us to confront this question directly.

The pion-nucleon coupling constants $g_{\pi N N}$ (CP conserving) and $\bar{g}_{\pi N N}$ (CP violating) are calculated in the large- $N_{c}$, three-flavour Skyrme model by direct evaluation of the leading matrix elements appearing in the LSZ reduction formula. We find that $g_{\pi N N} \sim N_{c}^{\frac{3}{2}}$, but that, at most, $\bar{g}_{\pi N N} \sim m_{\pi}^{2} N_{c}^{-\frac{1}{2}}$. These results show that the leading contribution to the neutron electric dipole moment in large- $N_{c}$ Skyrme model is the direct one $\left(D_{n} \sim N_{c} m_{\pi}^{2}\right)$, rather than the pion loop contribution.
\end{abstract}

\footnotetext{
${ }^{*}$ Supported in part by the DOE under grant DE-FG02-92ER40706
} 


\section{Introduction}

Some time ago one of us considered chiral fluctuations about a rotating, threeflavour skyrmion

$$
U(\mathbf{r}, t)=U_{\phi}(\mathbf{r}, t) A(t) U_{0}(\mathbf{r}) A^{-1}(t) U_{\phi}(\mathbf{r}, t)
$$

where $U_{0}(\mathbf{r})$ is a static solution of the $\mathrm{U}(3)$ Skyrme model, $A(t)$ is an $\mathrm{SU}(3)$ matrix of collective coordinates, and the matrix field (summation convention throughout)

$$
U_{\phi}=\exp \left\{\frac{i}{f_{\pi}} \phi_{\alpha} \lambda_{\alpha}\right\}
$$

is built from the nonet of pseudoscalar meson fields $\phi_{\alpha}(\alpha=0,1, \ldots, 8)$ and the Gell-Mann matrices $\lambda_{a}(a=1, \ldots, 8)$ with $\lambda_{0}=\sqrt{2 / 3}$ I. Insertion of expression 1.1 into the Skyrme lagrangian, $\mathcal{L}_{S}$, yields the elegant result

$$
\mathcal{L}_{S}(U(\mathbf{r}, t))=\mathcal{L}_{S}\left(A U_{0} A^{-1}\right)+\mathcal{L}_{\sigma}\left(U_{2 \phi}\right)+\mathcal{L}_{I}\left(A U_{0} A^{-1} ; \phi\right)
$$

in which the right-hand side is isomorphic to the low-energy soft-meson and baryon effective lagrangian

$$
\mathcal{L}_{\text {eff }}=\mathcal{L}_{B}(\psi)+\mathcal{L}_{\sigma}(\pi)+\mathcal{L}_{I}(\psi ; \pi)
$$

which describes free baryons interacting with the mesons of the non-linear $\sigma$-model. Since 1.4 obeys all the soft-meson theorems of chiral symmetry, one expects that these theorems are obeyed by the skyrmion-meson system of 1.3 as well.

The Skyrme lagrangian gives a formal result for the Yukawa couplings of the skyrmion-meson interaction. However, to arrive at a conclusive result for the Yukawa couplings involves a number of subtleties which lead to the central issues of this paper. Given that 1.3 includes appropriate CP violating terms, one can compute both the $\mathrm{CP}$ violating pion-nucleon scalar coupling constant $\bar{g}_{\pi N N}$ and the CP conserving pseudoscalar coupling constant $g_{\pi N N}$ appearing in the interaction term of eq. 1.4

$$
\mathcal{L}_{I}(\psi ; \pi)=\boldsymbol{\pi} \cdot \bar{\psi} \boldsymbol{\tau}\left(i \gamma_{5} g_{\pi N N}+\bar{g}_{\pi N N}\right) \psi
$$

Since the Skyrme model is formulated in terms of both chiral symmetry and the large $N_{c}$ (number of colors) expansion, one expects that its predictions for $g_{\pi N N}$ and $\bar{g}_{\pi N N}$ are consistent with QCD in the same limits. Naïvely, the dependence of the couplings on the pion (nucleon) mass $m_{\pi}\left(M_{N}\right)$ and the number of colors $N_{c}$ is, from 1.3 and ref. 11,

$$
g_{\pi N N} \sim N_{c}^{\frac{3}{2}} \quad \bar{g}_{\pi N N} \sim m_{\pi}^{2} N_{c}^{\frac{1}{2}} \quad M_{N} \sim N_{c} .
$$

Current algebra provides an ostensibly model-independent derivation of the leading contribution to the electric dipole moment of the neutron $D_{n}$ in the chiral limita

$$
D_{n}=\frac{g_{\pi N N} \bar{g}_{\pi N N}}{4 \pi^{2} M_{N}} \ln \left(\frac{M_{N}}{m_{\pi}}\right) .
$$


If eq. 1.6 were correct, the leading contribution in the combined chiral and large $N_{c}$ limit (and so dominant prediction of the Skyrme model in these limits) would be $D_{n} \sim N_{c} m_{\pi}^{2} \ln m_{\pi}$.

Recently significant progress in resolving this issue has been made in calculating $g_{\pi N N}$ (in the two flavour Skyrme model) by the authors of ref. 3 . It is the purpose of this paper to calculate the $\mathrm{CP}$ conserving and and $\mathrm{CP}$ violating $\pi-\mathrm{N}$ couplings $g_{\pi N N}$, and $\bar{g}_{\pi N N}$ by the methods of ref. 3, generalized to the three-flavour Skyrme model. We will show that although the pseudoscalar coupling $g_{\pi N N}$ indeed has the leading behaviour of eq. 1.6, the CP violating coupling $\bar{g}_{\pi N N}$ is down by a factor of at least $N_{c}^{-1}$ from that of eq. 1.6. Therefore, the pion contribution to $D_{n}$ is at most

$$
D_{n} \sim m_{\pi}^{2} \ln m_{\pi}
$$

and is therefore subdominant to the direct CP violating behaviour $D_{n} \sim m_{\pi}^{2} N_{c}$ computed in ref. 1 . (For a review see ref. 5.)

It is important to understand why there is an issue with regard to the Skyrme model Yukawa couplings. These couplings embody the linear fluctuations about the Skyrme classical solution. For constant collective coordinates ( $A=$ constant), this linear coupling must vanish for the classical solution to be stable under linear fluctuations. Thus the classical, linear meson-skyrmion interactions behave as

$$
\mathcal{L} \sim \phi(\dot{A} \text { terms })
$$

if the Skyrme equations of motion are imposed. The time derivatives in eq. 1.9 imply a suppression of at least a factor of $N_{c}^{-1}$ compared to the behaviour given in 1.6. If the chiral fluctuations are only considered classically, this would be the end of the story. However, the classical argument cannot be trusted since the chiral fluctuations must be quantized in order to properly account for the mesons as quantum fields. 6 , This is the origin of a number of complications.

The canonical momenta of the rotating skyrmion and associated mesons are not independent, and the separation into components transverse to the zero-mode solutions is "gauge dependent." Correctly calculating an $S$-matrix element-which must be gauge invariant - such as the $\pi$-N scattering amplitude is a complicated task. $\mathrm{B}$ The advance made by the authors of ref. 3 is to calculate the Born terms of $\pi$-N scattering in a manifestly gauge-invariant manner in the (two-flavour) Skyrme model. They obtain the same result as that given by the gauge-dependent separation of skyrmion and meson momenta, 8 confirming that the Yukawa coupling $g_{\pi N N}$ has the large $N_{c}$ behaviour of eq. 1.6. This suggests that the large- $N_{c}$ analysis of CP conserving $\pi$-N scattering in the Skyrme model is consistent with the implications of chiral symmetry.

The gauge-invariant calculation presented in ref. 3 is considerably simpler than the calculations in a gauge that separates the skyrmion and meson momenta. As a result, application of this method to the calculation of the $\pi-N$ couplings in the three-flavour Skyrme model yields a straightforward estimate of the large- $N_{c}$ behaviour of $g_{\pi N N}$ and $\bar{g}_{\pi N N}$. 


\section{The CP Violating Skyrmion}

In order to obtain strong $\mathrm{CP}$ violation, one must consider a $\mathrm{U}(3)$ non-linear $\sigma$ model so as to include $(i)$ an effective interaction representing the $\mathrm{U}(1)$ anomaly and (ii) a $\mathrm{CP}$ violating mass-matrix. The appropriate effective lagrangian in the large $N_{c}$ limit is then

$\mathcal{L}(U)=\mathcal{L}_{S}(U)+\frac{f_{\pi}^{2}}{16} \operatorname{Tr}\left(M U+M^{\dagger} U^{\dagger}-M-M^{\dagger}\right)+\frac{a f_{\pi}^{2}}{64 N_{c}}\left(\operatorname{Tr}\left[\ln U-\ln U^{\dagger}\right]\right)^{2}+\mathcal{L}_{W Z}$

where $\mathcal{L}_{W Z}$ is the Wess-Zumino 5 -form, $U$ is a $\mathrm{U}(3)$ matrix field, $a$ depends on the physical meson masses,

$$
\mathcal{L}_{S}(U)=\frac{f_{\pi}^{2}}{16} \operatorname{Tr}\left[\left(\partial_{\mu} U\right)\left(\partial_{\mu} U^{\dagger}\right)\right]+\frac{1}{32} e^{-2} \operatorname{Tr}\left\{\left[\left(\partial_{\mu} U\right) U^{\dagger},\left(\partial_{\nu} U\right) U^{\dagger}\right]^{2}\right\}
$$

and

$$
M=\operatorname{diag}\left[M_{1} \exp \left(i \frac{\beta_{1}}{M_{1}}\right), M_{2} \exp \left(i \frac{\beta_{2}}{M_{2}}\right), M_{3} \exp \left(i \epsilon \frac{\beta_{3}}{M_{3}}\right)\right] .
$$

In order to prevent strong $\mathrm{CP}$ violation from creating Goldstone bosons from the vacuum we requirel, 目

$$
\begin{aligned}
& \beta_{1}=\beta_{2}=\beta_{3} \equiv \beta \\
& \epsilon=\frac{a+M_{3}}{a+M_{1}}
\end{aligned}
$$

up to terms of $\mathcal{O}\left(\beta^{2}\right)$. It is convenient to define $\theta=\arg \operatorname{det} M$, the effective $\mathrm{CP}$ angle, and to set

$$
\theta=\lambda_{\theta}\left(\frac{1}{M_{1}}+\frac{1}{M_{2}}+\frac{\epsilon}{M_{3}}\right)
$$

so that $\lambda_{\theta}=\beta$.

The classical SU(3) Skyrme ansatz, neglecting CP violation, is 10, 11

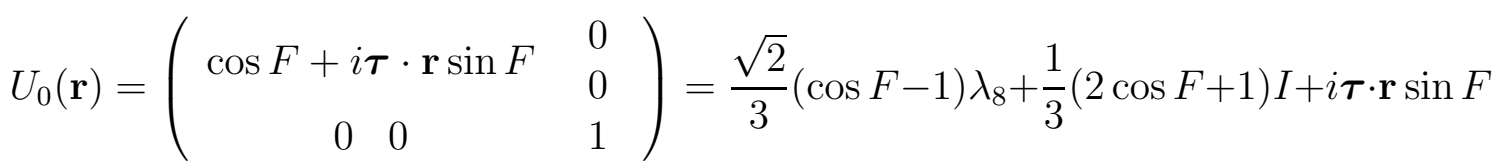

where $F=F(r)$. As emphasized by the authors of ref. 1 the $\mathrm{CP}$ violating term in $\mathcal{L}$ reacts back on the skyrmion. We adopt their generalization of ansatz 2.6 and consider

$$
U_{c}(\mathbf{r})=\exp \left(\frac{2 i}{f_{\pi}}\left[\sqrt{\frac{2}{3}}\left(f_{0}(r)-\delta\right) I+\lambda_{8} f_{8}(r)\right]\right) U_{0}(\mathbf{r})
$$

where

$$
\delta=\frac{\sqrt{6}}{4} \frac{\lambda_{\theta} f_{\pi}}{a+m_{\pi}^{2}}
$$


is the further vacuum shift required in order to prevent the creation of Goldstone bosons from the vacuum. The ansatz in eq. 2.7 remains spherically symmetric under combined isospin and spatial rotations. Neglecting isospin violation one can take

$$
M_{1}=M_{2}=m_{\pi}^{2} \quad M_{3}=2 m_{K}^{2}-m_{\pi}^{2} \quad 3 a / N_{c}=m_{\eta}^{2}+m_{\eta^{\prime}}^{2}-2 m_{K}^{2}
$$

so that $\lambda_{\theta} \sim \frac{1}{2} m_{\pi}^{2} \theta$. Since it does not affect any issues of principle, we neglect $\eta-\eta^{\prime}$ mixing and corrections of order $\mathcal{O}\left(M_{1} / a\right)$ in order to simplify the discussion. Taking these effects into account would only produce small numerical corrections of our results. The $\mathrm{CP}$ violating $(C P V)$ part of the lagrangian, which contains contributions from both the mass and anomaly terms, ${ }^{-1}$ provides a source for $f_{0}(r)$ and $f_{8}(r)$ :

$$
\delta \mathcal{L}_{C P V}=\frac{\lambda_{\theta} f_{\pi}}{2 \sqrt{3}}(1-\cos F)\left(f_{8}+\sqrt{2} f_{0}\right)+\mathcal{O}\left(\frac{M_{1}}{a}\right) .
$$

The function $F(r)$ is unchanged to order $\mathcal{O}(\theta)$ by the presence of this perturbation.

Neglecting terms of order $\mathcal{O}\left(m_{\pi}^{2} / m_{\eta}^{2}\right)$ one can use this source to solve for $f_{0}$ and $f_{8}$ in terms of $F(r)$. The result is

$$
\begin{aligned}
& f_{8}(r)=\frac{\lambda_{\theta} f_{\pi}}{2 \sqrt{3}} \int \frac{\mathrm{d}^{3} r^{\prime}}{4 \pi} \frac{e^{-m\left|\mathbf{r}-\mathbf{r}^{\prime}\right|}}{\left|\mathbf{r}-\mathbf{r}^{\prime}\right|}\left[1-\cos F\left(r^{\prime}\right)\right] \\
& f_{0}(r)=\frac{\sqrt{2} \lambda_{\theta} f_{\pi}}{2 \sqrt{3}} \int \frac{\mathrm{d}^{3} r^{\prime}}{4 \pi} \frac{e^{-m^{\prime}\left|\mathbf{r}-\mathbf{r}^{\prime}\right|}}{\left|\mathbf{r}-\mathbf{r}^{\prime}\right|}\left[1-\cos F\left(r^{\prime}\right)\right]
\end{aligned}
$$

where we have neglected $\eta-\eta^{\prime}$ mixing, and have written $m=m_{\eta}$ and $m^{\prime}=m_{\eta^{\prime}}$. The angular integration in eqs. 2.10 gives

$$
\begin{aligned}
& f_{8}(r)=\frac{\lambda_{\theta} f_{\pi}}{2 \sqrt{3} m} \int_{0}^{\infty} \mathrm{d} r^{\prime} G_{m}\left(r, r^{\prime}\right)\left[1-\cos F\left(r^{\prime}\right)\right] \\
& f_{0}(r)=\frac{\sqrt{2} \lambda_{\theta} f_{\pi}}{2 \sqrt{3} m^{\prime}} \int_{0}^{\infty} \mathrm{d} r^{\prime} G_{m^{\prime}}\left(r, r^{\prime}\right)\left[1-\cos F\left(r^{\prime}\right)\right]
\end{aligned}
$$

where

$$
G_{m}\left(r, r^{\prime}\right)= \begin{cases}\left(\frac{r^{\prime}}{r}\right) e^{-m r^{\prime}} \sinh (m r) & r<r^{\prime} \\ \left(\frac{r^{\prime}}{r}\right) e^{-m r} \sinh \left(m r^{\prime}\right) & r>r^{\prime}\end{cases}
$$

The contributions of $f_{8}(r)$ and $f_{0}(r)$ will be important in what follows.

\section{Meson-Nucleon Scattering in the Skyrme Model}

Here we generalize the analysis of ref. 3 to the CP-violating, three-flavour model described in the previous section. Denote the meson field (its quantum nature signaled by the absence of the subscript $c$ ) by

$$
U(\mathbf{r}, t)=\lambda_{\alpha} U_{\alpha} \quad \alpha=0,1, \ldots, 8 .
$$


It is convenient to write

$$
U_{\alpha}=s_{\alpha}+i p_{\alpha}
$$

where $s_{\alpha}$, and $p_{\alpha}$ are real fields. The non-linear $\sigma$ model constraint that $U$ is a $\mathrm{U}(3)$ field,

$$
U^{\dagger} U=I
$$

gives nine constraint equations. Therefore we can regard the $s_{\alpha}$ fields as dependent variables expressible in terms of the dynamical fields $p_{\alpha}$. (These nine independent real fields embody the pseudoscalar nonet.)

Substituting eq. 3.1 into the lagrangian (eq. 2.1) we obtain (exhibiting all time derivatives explicitly)

$$
\mathcal{L}=\frac{1}{2} \dot{p}_{\alpha} K_{\alpha \beta} \dot{p}_{\beta}+W_{\alpha} \dot{p}_{\alpha}-\mathcal{V}(p, \nabla p)
$$

where $\alpha, \beta=0,1, \ldots, 8$ and $W_{\alpha}$ is obtained from the Wess-Zumino term. (This is the analogue of eq. 3.3 of ref. 3.) In the weak field limit

$$
K_{\alpha \beta}=\frac{f_{\pi}^{2}}{4} \delta_{\alpha \beta}+\ldots
$$

Beyond this, the detailed forms of $K_{\alpha \beta}, W_{\alpha}$, and $\mathcal{V}(p, \nabla p)$ will not be needed. The canonical momenta are

$$
\Pi_{\alpha}=\frac{\delta L}{\delta \dot{p}_{\alpha}}=K_{\alpha \beta} \dot{p}_{\beta}+W_{\alpha}
$$

so that

$$
\dot{p}_{\alpha}=\left(K^{-1}\right)_{\alpha \beta}\left(\Pi_{\beta}-W_{\beta}\right) .
$$

Consequently, the Hamiltonian is, up to terms of $\mathcal{O}\left(\hbar^{2}\right)$ since we have ignored the problem of operator ordering,

$$
\mathcal{H}=\frac{1}{2} \int \mathrm{d}^{3} x(\Pi-W)_{\alpha}\left(K^{-1}\right)_{\alpha \beta}(\Pi-W)_{\beta}+\mathcal{V}(p, \nabla p)
$$

and the canonical commutation relations are

$$
\left[p_{\alpha}(x), \Pi_{\beta}(y)\right] \delta\left(x^{0}-y^{0}\right)=i \delta_{\alpha \beta} \delta^{(4)}(x-y)
$$

Due to the derivative couplings $K_{\alpha \beta} \neq \frac{f_{\pi}^{2}}{4} \delta_{\alpha \beta}$ and $\mathcal{H}_{\text {int }} \neq-\mathcal{L}_{\text {int }}$, as is evident from eq. 3.4 3.9. This is the essential reason that the classical argument for the absence of Yukawa couplings described in the introduction fails.

The skyrmion soliton is the static but rotated solution (i.e. , $U^{s}=A U_{c} A^{-1}$ ) to the equations of motion obtained from eq. 3.4. (The superscript $s$ will denote the static, classical part of a quantity in the following.) In terms of the field of eq. 2.7, this solution is given by

$$
p_{\alpha}^{s}=\frac{1}{2} \operatorname{Im} \operatorname{Tr}\left(\lambda_{\alpha} A U_{c} A^{-1}\right)
$$


where $A \in \mathrm{SU}(3)$ rotates the ansatz $U_{c}$ to an arbitrary direction in $\mathrm{SU}(3)$ space and for the static solution we neglect any time dependence of $A$.

In order to obtain the S-matrix for the meson-nucleon elastic scattering, we will analyze the LSZ reduction formula. To carry this out we must identify the interpolating field $\Phi_{\alpha}$. In fact, it is proportional to the quantum field $p_{\alpha}$ in eq. 3.4 (not the classical $p_{\alpha}^{s}$ ). Since $\lim _{r \rightarrow \infty} U_{c}(r)=\mathrm{I}$, we can pick out the proportionality constant from the non-linear meson fields which appear in eqs. $1.2 \& 1.3$ by using the fact that $\lim _{r \rightarrow \infty} U(\mathbf{r}, t)=U_{2 \phi}$ :

$$
U_{2 \phi} \sim 1+\frac{2 i}{f_{\pi}} \lambda \cdot \phi+\mathcal{O}\left(\frac{\phi^{2}}{f_{\pi}^{2}}\right)
$$

so that

$$
\Phi_{\alpha}=\frac{f_{\pi}}{2} p_{\alpha}
$$

Therefore

$$
\Phi_{\alpha}=\frac{f_{\pi}}{4} \operatorname{Im} \operatorname{Tr}\left[\lambda_{\alpha} U(\mathbf{r}, t)\right]
$$

where $U(\mathbf{r}, t)$ is the complete field $U_{\phi} A U_{c} A^{-1} U_{\phi}$ that enters $\mathcal{L}(U)$.

The reduction formula for our problem is given in terms of the source term $\mathcal{J}(x)$, $\Phi$ and $\dot{\Phi}$, as follows

$$
\begin{aligned}
& S_{f i}=\delta_{\mathrm{fi}}+i^{2} \int \mathrm{d}^{3} \mathbf{r}^{\prime} \mathrm{d} t^{\prime} \int \mathrm{d}^{3} \mathbf{r} \mathrm{d} t f_{k^{\prime}}^{*}\left(x^{\prime}\right) f_{k}(x) \\
& \quad \times\left\langle B^{\prime}\left(\mathbf{p}^{\prime}\right)\left|T\left(\mathcal{J}_{\beta}\left(x^{\prime}\right) \mathcal{J}_{\alpha}(x)\right)+\delta\left(t^{\prime}-t\right)\left[\dot{\Phi}_{\beta}\left(x^{\prime}\right), \mathcal{J}_{\alpha}(x)\right]-i \omega_{k^{\prime}} \delta\left(t^{\prime}-t\right)\left[\Phi_{\beta}\left(x^{\prime}\right), \mathcal{J}_{\alpha}(x)\right]\right| B(\mathbf{p})\right\rangle
\end{aligned}
$$

with $f_{\mathbf{k}}(x)=e^{-i k \cdot x} / \sqrt{(2 \pi)^{3} 2 \omega_{k}}$, where $k \cdot x=\omega_{k} t-\mathbf{k} \cdot \mathbf{r}$. The source term of the meson fields is

$$
\mathcal{J}_{\alpha}(x)=-\left[H,\left[H, \Phi_{\alpha}(x)\right]\right]+\left(-\nabla^{2}+m_{\pi}^{2}\right) \Phi_{\alpha}(x)
$$

with the commutator computed using eq. 3.8, 3.9, and 3.12. The result is

$$
\begin{aligned}
& {\left[H,\left[H, p_{\alpha}(y)\right]\right]=\left(K^{-1}\right)_{\alpha \beta} \frac{\delta \mathcal{V}(\mathbf{p}, \nabla p)}{\delta p_{\beta}}} \\
& +\frac{1}{2}(\Pi-W)_{\gamma}\left(K_{\alpha \beta}^{-1} K_{\gamma \delta, \beta}^{-1}-2 K_{\gamma \beta}^{-1} K_{\alpha \delta, \beta}^{-1}\right)(\Pi-W)_{\delta} \\
& +(\Pi-W)_{\gamma} K_{\alpha \beta}^{-1} K_{\gamma \delta}^{-1}\left(W_{\beta, \delta}-W_{\delta, \beta}\right)
\end{aligned}
$$

where for any quantity $T$ and any index $\alpha$, the notation $T_{, \alpha}$ denotes $\delta T / \delta p_{\alpha}$. We have not kept track of operator orderings, so that eq. 3.16 is accurate only up to terms of $\mathcal{O}\left(\hbar^{2}\right)$. We again see that the presence of derivative couplings in $\mathcal{L}$ and $\mathcal{H}$ produces a different result in eq. 3.16 than that expected from classical considerations. 
Similarly, using eq. $3.15 \& 3.16$, we find that

$$
\begin{aligned}
{\left[\dot{p}_{\beta}(x), \mathcal{J}_{\alpha}(y)\right] \delta\left(x^{0}-y^{0}\right)=} & -i\left(K^{-1}\right)_{\beta \gamma} \frac{\delta \mathcal{J}_{\alpha}(y)}{\delta p_{\gamma}(x)} \delta\left(x^{0}-y^{0}\right) \\
& +\left[\left(K^{-1}\right)_{\beta \gamma}, \mathcal{J}_{\alpha}(y)\right](\Pi-W)_{\gamma} \delta\left(x^{0}-y^{0}\right) \\
& -\left(K^{-1}\right)_{\beta \gamma}\left[W_{\gamma}(x), \mathcal{J}_{\alpha}(y)\right] \delta\left(x^{0}-y^{0}\right)
\end{aligned}
$$

with

$$
\left[\dot{\Phi}_{\beta}(x), \mathcal{J}_{\alpha}(y)\right] \delta\left(x^{0}-y^{0}\right)=\frac{f_{\pi}}{2}\left[\dot{p}_{\beta}(x), \mathcal{J}_{\alpha}(y)\right] \delta\left(x^{0}-y^{0}\right)
$$

We also need

$$
\left[\Phi_{\beta}(x), \mathcal{J}_{\alpha}(y)\right] \delta\left(x^{0}-y^{0}\right)=i \frac{f_{\pi}}{2} \frac{\delta \mathcal{J}_{\alpha}(y)}{\delta \Pi_{\beta}(x)} \delta\left(x^{0}-y^{0}\right)
$$

The above equations are again accurate only to order $\hbar$.

In order to evaluate the Born terms from eq. 3.14 we need certain one-baryon matrix elements, which will be analyzed in terms of the $1 / N_{c}$ expansion.

The crucial point is that in the matrix elements of operators taken between single-baryon states, one may replace the field operators with the classical fields evaluated with the skyrmion solution (3.10), i.e. , $p_{\alpha}(x) \rightarrow p_{\alpha}^{s}(x)$. This idea goes all the way back to reference 12 . The reason is that the fluctuating parts vanish since the "in" and "out" meson states form a Fock space on the one-baryon subspace. As emphasized in ref. 3 it is not necessary to explicitly seperate $\Phi_{\alpha}$ into static and fluctuating parts, since the one-baryon meson matrix elements will automatically pick out the static solution.

In order to arrange the one-baryon matrix elements in powers of $1 / N_{c}$, we need to order the various classical field quantities in terms of powers of $N_{c}$. By the classical equations of motion

$$
\frac{\delta \mathcal{V}\left(p^{s}, \nabla p^{s}\right)}{\delta p_{\alpha}^{s}}=0
$$

Note that the ordering in $1 / N_{c}$ is the same for the $\mathrm{CP}$ conserving (CPC) and $\mathrm{CP}$ violating $(\mathrm{CPV})$ terms in $p_{\alpha}^{s}$, to leading order in $\mathrm{CP}$ violation, as can be seen from the following. From eq. 3.10, the CP conserving term in $p_{\alpha}^{s}$ is

$$
\left(p_{\alpha}^{s}\right)_{\mathrm{CPC}}=\frac{1}{2} \operatorname{tr}\left(\lambda_{\alpha} A \boldsymbol{\tau} A^{-1}\right) \cdot \mathbf{r} \sin F(r)
$$

so that

$$
\left(p_{\alpha}^{s}\right)_{\mathrm{CPC}} \sim \mathcal{O}(1)
$$


in the $1 / N_{c}$ expansion. Then,

$$
\begin{aligned}
p_{\alpha}^{s} & =\left(p_{\alpha}^{s}\right)_{\mathrm{CPC}}+\left(p_{\alpha}^{s}\right)_{\mathrm{CPV}} \\
& \sim \mathcal{O}(1)\left[1+\lambda_{\theta} / m_{\eta}^{2}\right] \\
& \sim \mathcal{O}(1)\left[1+\left(\frac{m_{\pi}^{2}}{m_{\eta}^{2}}\right) \theta\right]
\end{aligned}
$$

where we have used eqs. 2.7 and 2.11. Therefore, the $1 / N_{c}$ estimates that follow hold in both the $\mathrm{CP}$ violating and conserving cases.

The estimates in eqs. 3.22 and 3.23 , and eq. 3.9 imply that

$$
\left(\Pi_{s}\right) \sim \mathcal{O}(1)
$$

Since

$$
\left(K_{s}\right)_{\alpha \beta} \sim \mathcal{O}\left(f_{\pi}^{2}\right) \sim \mathcal{O}\left(N_{c}\right)
$$

one also has

$$
\begin{array}{ll}
\dot{p}_{\alpha}^{s} & \sim \mathcal{O}\left(N_{c}^{-1}\right) \\
\left(K^{-1}\right)_{\alpha \beta}^{s} & \sim \mathcal{O}\left(N_{c}^{-1}\right) \\
\left(W^{s}\right)_{\alpha} & \sim \mathcal{O}(1)
\end{array}
$$

for these classical quantities. With these ingredients we can now make $1 / N_{c}$ estimates of the one-baryon matrix elements.

For the first term in eq. 3.14 we need

$$
\begin{aligned}
\left\langle B\left|\mathcal{J}_{\alpha}(x)\right| B^{\prime}\right\rangle & =\left\langle B\left|\left(-\nabla^{2}+m_{\pi}^{2}\right) \Phi_{\alpha}+\partial_{t}^{2} \Phi_{\alpha}\right| B^{\prime}\right\rangle \\
& =\frac{f_{\pi}}{2}\left\{\left\langle B\left|\left(-\nabla^{2}+m_{\pi}^{2}\right)\left(p_{s}\right)_{\alpha}\right| B^{\prime}\right\rangle+\mathcal{O}\left(1 / N_{c}^{2}\right)\right\}
\end{aligned}
$$

which holds because all the terms in eq. 3.16, evaluated with the classical solution, contribute at most to the order $\mathcal{O}\left(1 / N_{c}^{2}\right)$ correction.

Similarly, from eq. 3.19

$$
\begin{aligned}
\left\langle B\left|\left[\Phi_{\beta}(x), \mathcal{J}_{\alpha}(y)\right] \delta\left(x^{0}-y^{0}\right)\right| B^{\prime}\right\rangle & =i \frac{f_{\pi}}{2}\left\langle B\left|\left[\frac{\delta \mathcal{J}_{\alpha}(y)}{\delta \Pi_{\beta}(x)}\right]_{s}\right| B^{\prime}\right\rangle \delta\left(x^{0}-y^{0}\right) \\
& \sim \mathcal{O}\left(f_{\pi}^{-3}\right) \sim \mathcal{O}\left(N_{c}^{-\frac{3}{2}}\right)
\end{aligned}
$$

and from eq. 3.18

$$
\begin{aligned}
\left\langle B\left|\left[\dot{\Phi}_{\beta}(x), \mathcal{J}_{\alpha}(y)\right] \delta\left(x^{0}-y^{0}\right)\right| B^{\prime}\right\rangle & =\frac{f_{\pi}}{2}\left\{-i\left\langle B\left|\left(K_{s}^{-1}\right)_{\beta \gamma}\left[\frac{\delta \mathcal{J}_{\alpha}(y)}{\delta p_{\gamma}(x)}\right]_{s}\right| B^{\prime}\right\rangle \delta\left(x^{0}-y^{0}\right)+\mathcal{O}\left(1 / N_{c}^{2}\right)\right\} \\
& \sim \mathcal{O}(1)
\end{aligned}
$$


Therefore only eq. 3.27 and 3.29 contribute to the Born terms in the large $N_{c}$ limit. In addition, only equation 3.27 appears in the pole term, and so gives both the $\mathrm{CP}$ conserving and $\mathrm{CP}$ violating Yukawa couplings, while eq. 3.29 contributes to a non-pole contact term.

\section{The $\pi$-N Coupling Constants}

The classical, CP-violating solution is, from eqs. 2.7, 3.12, and 3.13,

$$
\left(\Phi_{\alpha}^{s}\right)_{\mathrm{CPV}}=\frac{1}{30 \sqrt{3}}\left\{\left[\sqrt{2}\left(f_{0}(r)-\delta\right)+f_{8}(r)\right](\cos F(r)-1)+4 f_{8}(r)\right\} \operatorname{Tr}\left(\lambda_{\alpha} A \lambda_{8} A^{-1}\right)
$$

Therefore, to leading order in $1 / N_{c}$,

$$
\begin{aligned}
\bar{g}_{\pi N N}(\mathbf{k}) & =\int \frac{\mathrm{d}^{3} r}{(2 \pi)^{3}} e^{-i \mathbf{k} \cdot \mathbf{r}}\left\langle B\left|\left(\mathcal{J}_{\alpha}\right)_{\mathrm{CPV}}\right| B^{\prime}\right\rangle \\
& \simeq \int \frac{\mathrm{d}^{3} r}{(2 \pi)^{3}} e^{-i \mathbf{k} \cdot \mathbf{r}}\left\langle B\left|\left(-\nabla^{2}+m_{\pi}^{2}\right)\left(\Phi_{\alpha}^{s}\right)_{\mathrm{CPV}}\right| B^{\prime}\right\rangle
\end{aligned}
$$

If the physical (mass-shell) coupling

$$
\bar{g}_{\pi N N}=\left.\bar{g}_{\pi N N}(\mathbf{k})\right|_{\mathbf{k}^{2}=-m_{\pi}^{2}}
$$

is to have a contribution from eq. 4.2, then $\left(\Phi_{\alpha}^{s}\right)_{C P V}$ must have a pion tail (i.e. it must behave as $\exp \left(-m_{\pi} r\right) / r$ as $\left.r \rightarrow \infty\right)$. Since

$$
F(r) \longrightarrow C \frac{e^{-m_{\pi} r}}{r} \text { as } r \rightarrow \infty
$$

it is clear that the $\cos F(r)$ factor does not have a pion tail. Further, eq. 2.10 shows that $f_{8}(r)$ and $f_{0}(r)$ have (momentum space) poles at $m_{\eta}^{2}$ and $m_{\eta^{\prime}}^{2}$, respectively, rather than at $m_{\pi}^{2}$, so that the required pion tail is entirely absent from eq. 4.1. By contrast, the $\mathrm{CP}$ conserving coupling is given by (the analysis is essentially the same as that already discussed in ref. 3)

$$
g_{\pi N N}(\mathbf{k})=\frac{2}{3} M_{N} f_{\pi} \frac{\mathbf{k}^{2}+m_{\pi}^{2}}{|\mathbf{k}|} \int \mathrm{d}^{3} r j_{1}(k r) \sin F(r)
$$

where $j_{1}(y)$ is the spherical Bessel function. Since $\sin F(r)(\sim F(r)$ as $r \rightarrow \infty)$ does have the required pion tail, $g_{\pi N N}$ is non-vanishing on-shell.

Therefore we conclude that $\bar{g}_{\pi N N}$ is suppressed relative to eq. 1.6 by at least a factor of $N_{c}^{-1}$, while the behaviour of $g_{\pi N N}$ is correctly given by 1.6.' It is important

\footnotetext{
${ }^{\dagger}$ Although the order $\mathcal{O}\left(N^{\frac{1}{2}}\right)$ contribution to $\bar{g}_{\pi N N}$ does not vanish off-shell, it is suppressed by the factor $m_{\pi}^{2} / m_{\eta}^{2}$, as is evident from eq. 3.23 .
} 
to note that since $g_{\pi N N}$ is not suppressed by factors of $N_{c}^{-1}$, the stability of the linear fluctuations about the classical solution cannot be the reason for the suppression of $\bar{g}_{\pi N N}$. The correct explanation rests with the asymptotic behaviour of the skyrmion profile function eq. 2.6, where one should compare the coefficient of $\lambda_{8}$ with that of $\tau$.

\section{Conclusions}

Our calculation shows that the Yukawa couplings in the Skyrme model involve issues more subtle than the vanishing of linear fluctuations about the classical solution. The CP conserving coupling $g_{\pi N N}$ is not suppressed, while the CP violating coupling is. Although this supports the final result of ref. \&, we find that neglect of the pion contribution to $D_{n}$ in the large $N_{c}$ limit requires more analysis than indicated there before one can be assured that the direct contribution to $D_{n}$ dominates. The behaviour of the CP violating coupling constant $\bar{g}_{\pi N N}$ is also interesting for other applications.13

The primary value of the Skyrme model is as a phenomenological approximation to the low-energy effective lagrangian of QCD; therefore, one hopes that it substantially embodies the physics of chiral symmetry and the large- $N_{c}$ properties of QCD. In fact, we have found that simple QCD estimates based on naïve quark-line counting (i.e. estimates similar to those of ref. 14 but using the $\theta g^{2} F \widetilde{F}$ interaction) agree with our Skyrme model results. In regard to this, it would have been peculiar if the Skyrme model, which does give the correct large $N_{c}$ behaviour for the multi-meson skyrmion, did not, in the end, give the correct Yukawa couplings as well.

\section{References}

[1] H. J. Schnitzer, Phys. Lett. B139 (1984) 217.

[2] V. Baluni, Phys. Rev. D19 (1979) 2227; R. Crewther, P. Di Vecchia, G. Veneziano, and E. Witten, Phys. Lett. B88 (1979) 123; errata B91 (1980) 487.

[3] A. Hayashi, S. Saito, and M. Uehara, Phys. Rev. D11 (1992) 4856.

[4] L. Dixon, A. Larignau, Y. Nir, and B. Warr, Phys. Lett. B253 (1991) 459.

[5] Y. Nir, "CP Violation," SLAC preprint, SLAC-PUB-5874, September 1992.

[6] H. J. Schnitzer, Nucl. Phys. B261 (1985) 546.

[7] H. J. Schnitzer, Phys. Lett. B253 (1991) 465. 
[8] A. Hayashi, S. Saito, and M. Uehara, Phys. Lett. B246 (1990) 15; Phys. Rev. D43 (1991) 1520; M. Uehara, A. Hayashi, and S. Saito, Nucl. Phys. A534 (1991) 680.

[9] C. Rosenzweig, J. Schechter, and C. Trahern, Phys. Rev. D21 (1980) 3388; E. Witten, Ann. Phys. 128 (1980) 363; P. Di Vecchia, F. Nicodemi, R. Pettorino, and G. Veneziano, Nucl. Phys. B181 (1981) 318.

[10] G. Adkins, C. Nappi, and E. Witten, Nucl. Phys. B228 (1983) 552; G. Adkins and C. Nappi, Nucl. Phys. B233 (1984) 109.

[11] E. Guadagnini, Nucl. Phys. B236 (1984) 35.

[12] J. Goldstone and R. Jackiw, Phys. Rev. D11 (1975) 2943. J.-L. Gervais, A. Jevicki, and B. Sakita, Phys. Rev. D12 (1975) 1038. E. Tomboulis, Phys. Rev. D12 (1975) 1678.

[13] W. Fischler, S. Paban, and S. Thomas, Phys. Lett. B289 (1992) 373.

[14] H. Müther, C. Engelbrecht, and G. Brown, Nucl. Phys. A462 (1987) 701. 